\title{
Interrogation Sensing Scheme Based on a Figure-of-Eight Fiber Loop Mirror
}

\author{
Ricardo M. Silva, A. Layeghi, M. I. Zibaii, Hamid Latifi, José Luís Santos, \\ Antonio Barbosa Lobo Ribeiro, and Orlando Frazão
}

\begin{abstract}
A fiber optic interrogation sensor scheme based on a "figure-of-eight" configuration created from a single directional $3 \times 3$ fiber optic coupler is proposed. Two loops are formed in each arm and one of them contains the sensing head and the other is used as reference signal. A theoretical study based on Jones matrix analysis of this fiber loop mirror combination is reported. The optical configuration is tested as an interrogation scheme for a fiber strain sensor where the spectral response arises from the combination of the reference signal modulated by the sensor signal. The strain sensor configuration shows a phase sensitivity of $6.7 \pm 4.38 \times 10^{-2} \mathrm{mrad} / \mu$ strain by linear regression.
\end{abstract}

Index Terms-Fiber interferometer, figure-of-eight, highlybirefringent fiber, optical fiber sensor, strain sensor.

\section{INTRODUCTION}

$\mathbf{F}$ IBER loop mirrors (FLM) are attractive devices to be used in sensing applications [1]-[3]. On a standard FLM, two output ports of $2 \times 2$ fiber coupler are spliced together to form a loop, and thus, all light is reflected back to the input port of the coupler. FLMs can incorporate within the loop a length of highly-birefringent $(\mathrm{HiBi})$ fiber, which reveals several advantages, such as, input polarization independence and high extinction ratio. Besides the gyroscope application [1], various kinds of fiber optic sensors based on FLMs have been demonstrated, such as temperature [4], [5], strain [6]-[8], pressure [9], liquid level [10], biochemical [11], UV [12] and multiparameter sensor [13], [14].

In 1999, a strain sensor based on a Hi Bi fiber Sagnac ring configuration was investigated, using a frequency modulated continuous wave [6]. Frazao et al. proposed a solution for simultaneous measurement of strain and temperature,

Manuscript received November 26, 2012; revised February 4, 2013; accepted February 19, 2013. Date of publication February 26, 2013; date of current version March 29, 2013.

R. M. Silva is with the Department of Physics and Astronomy, Faculty of Sciences, University of Porto, Porto 4169-007, Portugal, and also with INESC TEC - Instituto de Engenharia de Sistemas e Computadores do Porto, Porto 4169-007, Portugal (e-mail: rmsilva@inescporto.pt).

A. Layeghi, M. I. Zibaii, and H. Latifi are with the Laser and Plasma Research Institute, Shahid Beheshti University, Tehran 1983963113, Iran (e-mail: a.layeghi@gmail.com; mizibaye@gmail.com; latifi@cc.sbu.ac.ir).

J. L. Santos is with the Department of Physics and Astronomy, Faculty of Sciences, University of Porto, Porto 4169-007, Portugal (e-mail: josantos@ fc.up.pt).

A. B. L. Ribeiro is with the Faculty of Heath Sciences, University Fernando Pessoa, Porto 4200-150, Portugal (e-mail: alobo@ufp.edu.pt).

O. Frazão is with INESC TEC - Instituto de Engenharia de Sistemas e Computadores do Porto, Porto 4169-007, Portugal (e-mail: ofrazao@inescporto.pt).

Color versions of one or more of the figures in this letter are available online at http://ieeexplore.ieee.org.

Digital Object Identifier 10.1109/LPT.2013.2248708

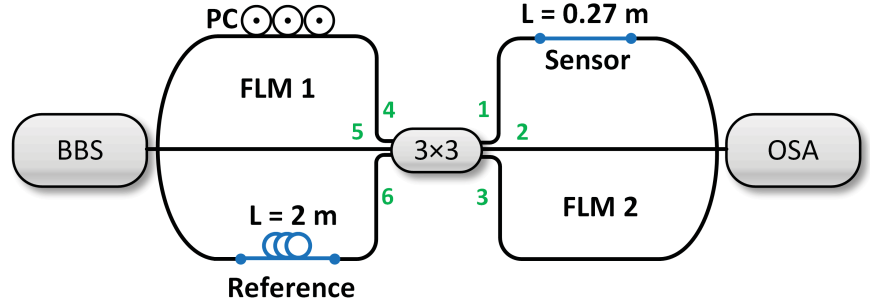

Fig. 1. Schematic of the experimental setup (BBS: broadband source; OSA: optical spectrum analyzer; PC: polarization controller; FLM 1,2: fiber loop mirror 1 and 2).

using a long period fiber grating (LPG) combined with a Hi Bi FLM [7]. A fiber-optic strain sensor was obtained by inserting a short length of PCF in a Hi Bi FLM [8]. Recently, three different high birefringent fiber loop mirrors (HiBi FLM) were analyzed both theoretically and experimentally [15]. All these three configurations can be applied in specific sensing applications; however one of them can be used simultaneously as a sensor and as an interrogation system by proper design and signal processing implementation.

This letter presents a theoretical and experimental study of a newly FLM based on a "figure-of-eight" configuration, constructed from a single directional $3 \times 3$ fiber optic coupler. The configuration was tested as an interrogation system for a fiber strain sensor, where the spectral response arises from the combination of the reference signal modulated by the sensor signal.

\section{EXPERIMENTAL DETAILS}

In Fig. 1 is shown a schematic of the proposed configuration to operate as an interrogation system for strain measurement. The experimental setup consisted on a broadband depolarised optical source based on erbium-doped fiber with a spectral bandwidth of $100 \mathrm{~nm}$ (FWHM) centered at $1550 \mathrm{~nm}$, a $3 \times 3$ fiber optic coupler in standard single mode fiber and an optical detection unit. The polarization controller is used only to optimize the visibility of the fringe pattern in each FLMs. In this case, an optical spectrum analyzer (OSA) is used to read the spectral peaks generated by the configuration. Two discrete FLMs (FLM1, FLM2) are formed in each side of the $3 \times 3$ coupler. For the case, the (FLM1) is used as reference loop signal with a total length of $3.5 \mathrm{~m}$ and the (FLM2) as sensing head with a total length of $1.5 \mathrm{~m}$. In order to construct both FLMs, two different lengths of HiBi fiber were spliced within the loops. The HiBi fiber used was an 
e-core fiber (produced by KHV Company) with a physical length of $1.5 \mathrm{~m}$ and $0.27 \mathrm{~m}$ for the reference (FLM1) and sensor (FLM2) loops respectively, with a group birefringence of $3.85 \times 10^{-4}$ and an optical loss of $9 \mathrm{~dB} / \mathrm{km}$. As seen in Fig. 1, the broadband source (BBS) at the input port (2) illuminates the configuration and light is divided into three beams by a 100/3-power ratio distributed by the three output ports $(1,2,3)$. According to the experimental setup on the FLM2, two light beams propagate in clockwise (from port-1) and counter-clockwise (from port-3) directions, respectively. When, these beams arrive the coupler, they split again into three beams which are divided by the 3 -input ports. The light beams from the input ports 4 and 6 counter-propagate each other through the FLM1, and then when arrive to the coupler again to induce the interference pattern.

The optical characteristics of these interference beams are determined by the birefringence of the HiBi fiber itself and by the splicing angle rotation of $\mathrm{HiBi}$ fiber axis (or the polarization state set on the PC on Fig. 1) simultaneously. To summarize, this optical configuration is similar to two fiber loops in series.

\section{TheORETICAL ANALYSIS}

In order to perform the theoretical analysis of the spectral transmitted signal, the Jones matrix formalism was used. The schematic of this new HiBi FLM interferometer is exhibited in Fig. 1. To simplify the analysis, it was assumed that the excess loss of the $3 \times 3$ fiber optic coupler is negligible for the fundamental mode propagation. It was also supposed that the coupling effect of the fiber optic coupler is sufficiently localized such the physical length of the coupling section can be insignificant. This simplification allowed excluding the longitudinal field variations before and after the coupling region.

It was considered the propagation direction of light in the configuration as shown in Fig. 2, taking into account the explanation described before. This interferometer has different interference periods due to the HiBi fiber lengths spliced inside of each loop. Using a similar method to our previous analysis of the transmission field [15], we can derive Jones matrix analysis of the transmission electric field ( $\left.E_{\text {Trans }}\right)$, expressed as

$$
E_{\text {Trans }}=\left(\begin{array}{l}
\left\{\begin{array}{l}
\left(\left[K_{42}\right]\left[R_{\theta 2}\right]\left[J_{H B 2}\right][R]\left[K_{36}\right]\right)+ \\
\left(\left[K_{62}\right][R]\left[J_{H B 2}\right]\left[R_{\theta 2}\right]\left[K_{34}\right]\right) \\
\times\left([R]\left[J_{H B 1}\right]\left[R_{\theta 1}\right]\left[K_{24}\right]\right)+ \\
\left\{\left(\left[K_{42}\right]\left[R_{\theta 2}\right]\left[J_{H B 2}\right][R]\left[K_{16}\right]\right)+\right. \\
\left(\left[K_{62}\right][R]\left[J_{H B 2}\right]\left[R_{\theta 2}\right]\left[K_{14}\right]\right) \\
\times\left(\left[R_{\theta 1}\right]\left[J_{H B 1}\right][R]\left[K_{52}\right]\right)
\end{array}\right\}
\end{array}\right)
$$

where, $\left[K_{i j}\right]$ is the coupling matrix of the $3 \times 3$ fiber coupler. In an ideal $3 \times 3$ fiber coupler, there is a $2 \pi / 3$ phase difference between any two beams of the three ports. The average phase differences between two of the three outputs measured experimentally confirms that assumption. These matrices are

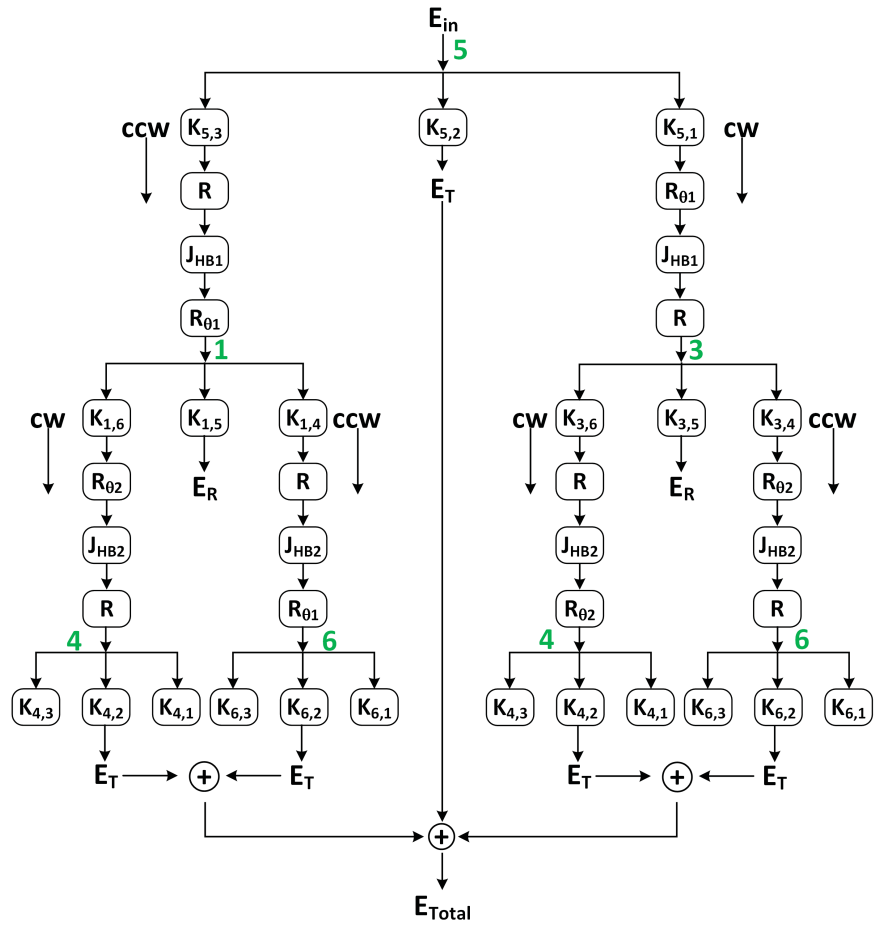

Fig. 2. Equivalent optical circuit of the proposed two fiber loops interferometer based on the Hi-Bi fibers.

given by:

$$
\begin{aligned}
& {\left[K_{41}\right]=\left[K_{42}\right]=\left[K_{43}\right]=\left[K_{51}\right]=\left[K_{61}\right]=\frac{1}{\sqrt{\alpha}}\left[\begin{array}{ll}
1 & 0 \\
0 & 1
\end{array}\right]} \\
& {\left[K_{53}\right]=\left[K_{62}\right]=\frac{e^{-i 2 \pi / 3}}{\sqrt{\beta}}\left[\begin{array}{ll}
1 & 0 \\
0 & 1
\end{array}\right]} \\
& {\left[K_{52}\right]=\left[K_{63}\right]=\frac{e^{i 2 \pi / 3}}{\sqrt{\gamma}}\left[\begin{array}{ll}
1 & 0 \\
0 & 1
\end{array}\right]}
\end{aligned}
$$

It is clear $K_{i j}=K_{j i}$. That $\alpha, \beta$ and $\gamma$ are the splitting power ratio of coupler for an asymmetric coupler. It is assumed $\beta=\gamma=2.94$ and $\alpha=5.125$, which are approximately according to experimental results of $3 \times 3$ fiber coupler used, while in an symmetric coupler, $\alpha, \beta$ and $\gamma$ are equal.

The matrix $[R]$ is a coordinate conversion matrix due to the folded fiber in the loop, and it is given by:

$$
[R]=\left[\begin{array}{cc}
-1 & 0 \\
0 & 1
\end{array}\right]
$$

Next, $\left[J_{H B}\right]$ is the Jones matrix of the HiBi fiber, which is expressed as

$$
\left[J_{H B}\right]=\left[\begin{array}{cc}
e^{i \beta_{e} L} & 0 \\
0 & e^{i \beta_{o} L}
\end{array}\right]
$$

Here, the propagation constants along the e-axis and o-axis of the HiBi fibers are represented by $\beta_{e}$ and $\beta_{o}$, respectively, and the length of the HiBi fiber is expressed as $L$. Based on the birefringence of the $\mathrm{HiBi}$ fiber, that is, $n_{e o}=n_{e}-n_{o}$, the difference of propagation constants, $\left(\beta_{e}-\beta_{o}\right)$, is equal to $\left(2 \pi n_{e o} / \lambda\right)$. The last $\left[R_{\theta}\right]$ is the Jones rotation matrix. This matrix is expressed as

$$
\left[R_{\theta}\right]=\left[\begin{array}{cc}
\cos \theta & \sin \theta \\
-\sin \theta & \cos \theta
\end{array}\right]
$$




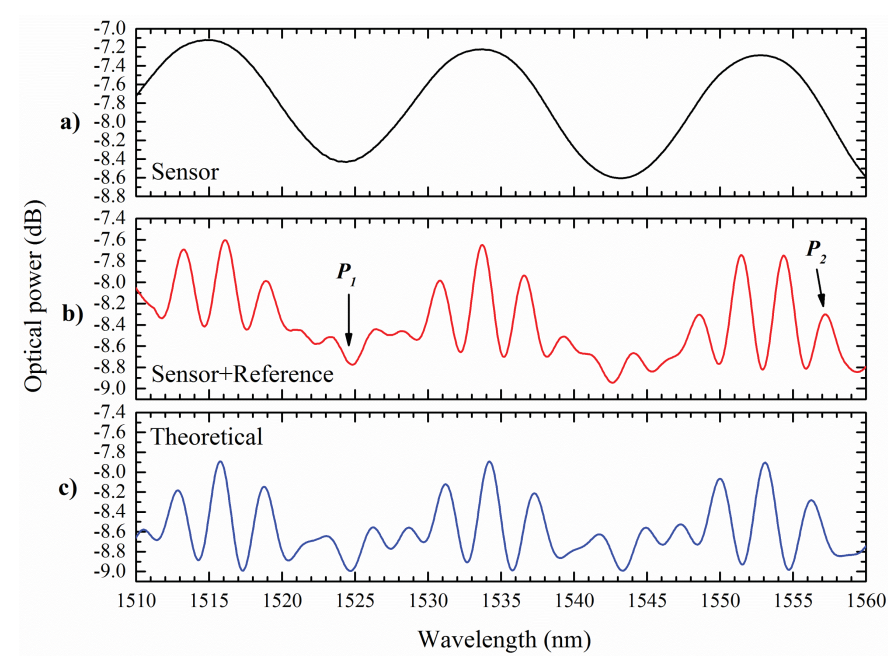

Fig. 3. Theoretical and experimental response of the sensing head.

where, $\theta$ is the rotation angle of the state of polarization with respect to the laboratory coordinates.

\section{EXPERIMENTAL RESULTS}

In Fig. 3 (a) it is exhibited the sensor loop spectral response. This signal corresponds only to the HiBi fiber length of $0.27 \mathrm{~m}$ spliced in the FLM2, wherein the HiBi fiber in FLM1 was not inserted in the experimental configuration. The sensor wavelength periodicity was $\sim 17 \mathrm{~nm}$.

The spectral response of the sensor signal combined with the reference signal when a second HiBi fiber length of nearly $1.5 \mathrm{~m}$ was spliced in FLM1 is shown in Fig. 3 (b). Two different frequencies are observed. The higher frequency corresponds to the reference signal and has a wavelength periodicity of $3 \mathrm{~nm}$. The lower frequency, shown from the amplitude modulation of the reference signal, was due to the sensor response. Fig. 3 (c) shows the theoretical simulation based on the model described before.

For mechanical strain characterization, the sensing head was attached to a translation stage with a step movement precision of $1 \mu \mathrm{m}$. All measurements were made at room temperature. An amplitude variation of the reference signal can be observed due to the fringe pattern wavelength shift of the sensor when strain was applied. Peaks $P_{1}$ and $P_{2}$ evidenced in the Fig. 3 (b) were nearly in quadrature. Using the variation of two peaks, it was possible to reconstruct the fringe pattern of the sensor signal [16]. The normalized amplitude outputs represented in Fig. 4 were obtained from both peaks. Besides the amplitude variation of the peaks when strain was applied, their curve fittings are also represented. The fit behavior is similar to a trigonometric function and is given by

$$
P_{n}=A \times \sin \left[\frac{\pi\left(x-x_{0}\right)}{f}\right]
$$

where, $A$ is the amplitude, $x$ is the applied strain, $x_{0}$ is a constant for initial $x$ and $f$ is the frequency.

Table 1, shows the values obtained from the fitting for the two peaks. It is observed that the parameter $f$ as almost the

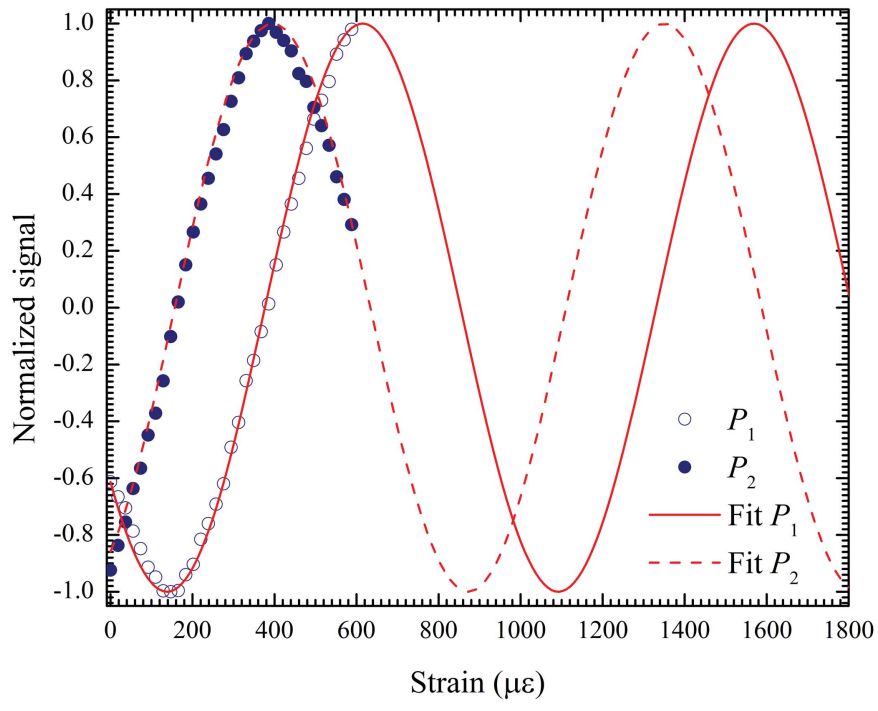

Fig. 4. Normalized output signals versus applied mechanical strain, and the correspondent curve fitting of the experimental data.

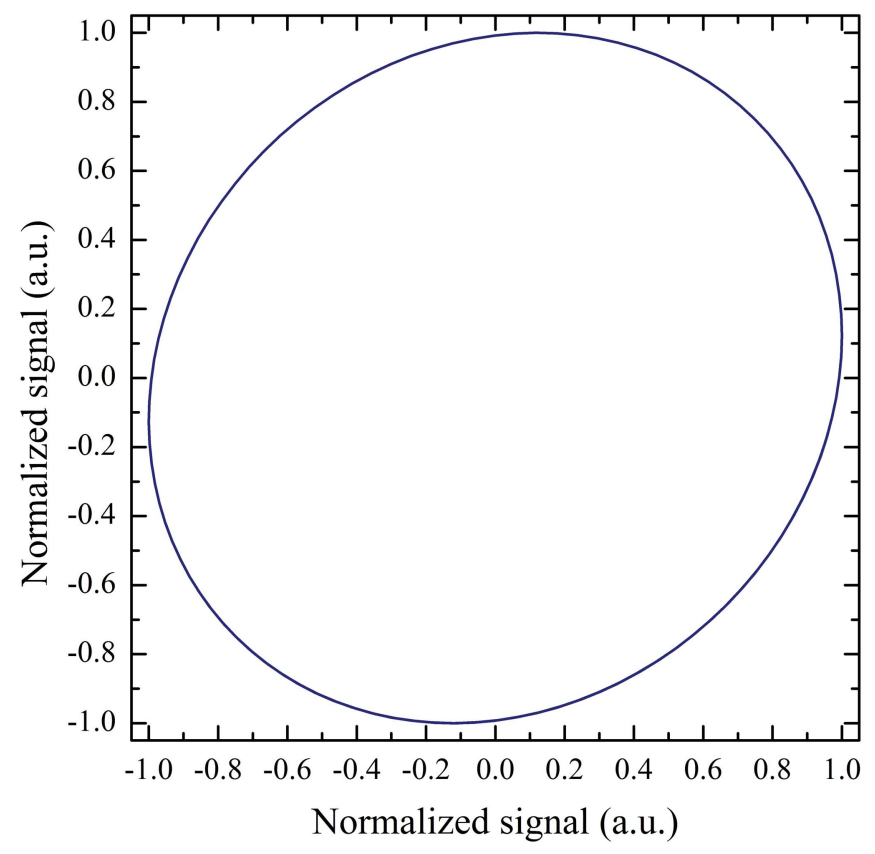

Fig. 5. $X-Y$ signal representation of both signals $\left(P_{1}\right.$-vertical axis and $P_{2}$-horizontal axis), illustrating the quadrature condition.

same value for the two peaks informing us that these are almost in quadrature. Fig. 5 reveals nearly that condition, which reveals that the system is operating close to ideal quadrature condition.

In this case, a nearly quadrature phase-shifted relation can be observed between these two signals, from which could be recover the optical phase through the relation:

$$
\varphi=\tan ^{-1}\left(P_{1} / P_{2}\right)
$$

where, $P_{1}$ and $P_{2}$ are the normalized optical powers, respectively [16]. A mechanical strain from 0 and $600 \mu$ strain was 
TABLE I

PARAmETERS ObTAINED From FitTING THE EXPERIMENTAL DATA

\begin{tabular}{|c|c|c|}
\hline & $\boldsymbol{P}_{\mathbf{1}}$ & $\boldsymbol{P}_{\mathbf{2}}$ \\
\hline $\boldsymbol{A}$ & 1 & 1 \\
\hline $\boldsymbol{x}_{\mathbf{c}}$ & 156.75 & 376.6 \\
\hline $\boldsymbol{f}$ & 477 & 483 \\
\hline
\end{tabular}

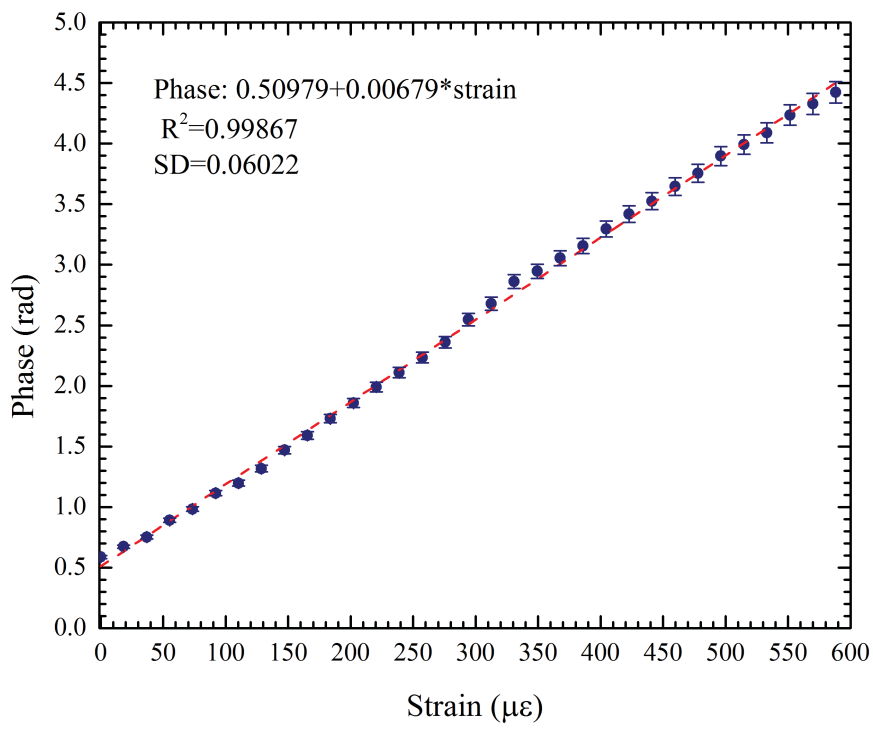

Fig. 6. Strain measurement using the phase recovery and the errors bars with $5 \%$ of the source errors.

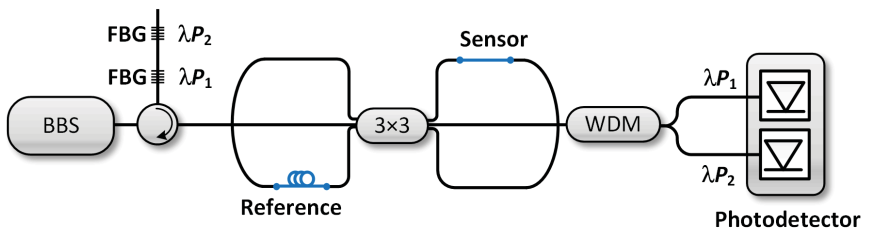

Fig. 7. Schematic diagram of a low cost interrogation system for possible application.

applied through the translation stage, resulting in a linear curve fitting slope correspondent to a phase-to-strain sensitivity of $6.7 \pm 4.38 \times 10^{-2} \mathrm{mrad} / \mu$ strain. The resultant induced phase change calculated by relation (7) is shown in Fig. 6. Due to the reason that the arctangent function signal is confined to the range of $\pm \pi / 2$ (limiting the unambiguous measurement range), a simple phase unwrapping algorithm can be applied to solve this limitation [17], by adding or subtracting $\pi$ to the extracted phase when reaches $\pm \pi / 2$ and changes abruptly in the amount of $\pm \pi$. Also, this $3 \times 3$ FLM sensor topology is temperature independent as shown in [18].

In Fig. 7, is presented the proposed low-cost interrogation system for the $\mathrm{HiBi}$ strain sensor, avoiding this way the optical spectrum analyzer unit of Fig. 1. The main components for the proposed system are a BBS, an optical circulator, two fiber Bragg gratings (with their center wavelengths tuned to the peaks desired), a wavelength-division multiplexing (WDM) fiber coupler and two photodetectors.

\section{CONCLUSION}

To summarize, a theoretical and experimental study for a fiber loop mirror based on a "figure-of-eight" made of a $3 \times 3$ fiber coupler, is demonstrated. The theoretical model based on Jones matrix formalism was studied and presents similar behavior when compared with the experimental fringe pattern. The configuration was tested as an interrogation system where the spectral response arises from the combination of the reference signal modulated by the sensor signal. The configuration was characterized in mechanical strain and presents a phase sensitivity of $6.7 \pm 4.38 \times 10^{-2} \mathrm{mrad} / \mu$ strain by linear regression.

\section{REFERENCES}

[1] B. Culshaw, "The optical fibre Sagnac interferometer: An overview of its principles and applications," Meas. Sci. Technol., vol. 17, no. 1, p. R1, Nov. 2005.

[2] Y. Liu, et al., "High-birefringence fiber loop mirrors and their applications as sensors," Appl. Opt., vol. 44, no. 12, pp. 2382-2390, Apr. 2005.

[3] D. B. Mortimore, "Fiber loop reflectors," J. Lightw. Technol., vol. 6, no. 7, pp. 1217-1224, Jul. 1988.

[4] E. DelaRosa, L. A. Zenteno, A. N. Starodumov, and D. Monzon, "All-fiber absolute temperature sensor using an unbalanced highbirefringence Sagnac loop," Opt. Lett., vol. 22, no. 7, pp. 481-483, Apr. 1997.

[5] A. N. Starodumov, L. A. Zenteno, D. Monzon, and E. DeLaRosa, "Fiber Sagnac interferometer temperature sensor," Appl. Phys. Lett., vol. 70, no. 1, pp. 19-21, Jan. 1997.

[6] M. Campbell, G. Zheng, A. S. Holmes-Smith, and P. A. Wallace, "A frequency-modulated continuous wave birefringent fibre-optic strain sensor based on a Sagnac ring configuration," Meas. Sci. Technol., vol. 10, no. 3, pp. 218-224, Mar. 1999.

[7] O. Frazäo, L. M. Marques, S. Santos, J. M. Baptista, and J. L. Santos, "Simultaneous measurement for strain and temperature based on a longperiod grating combined with a high-birefringence fiber loop mirror," IEEE Photon. Technol. Lett., vol. 18, no. 22, pp. 2407-2409, Nov. 15, 2006.

[8] X. Y. Dong, H. Y. Tam, and P. Shum, "Temperature-insensitive strain sensor with polarization-maintaining photonic crystal fiber based Sagnac interferometer," Appl. Phys. Lett., vol. 90, no. 15, pp. 151113-1-151113-3, Apr. 2007.

[9] H. Fu, et al., "Pressure sensor realized with polarization-maintaining photonic crystal fiber-based Sagnac interferometer," Appl. Opt., vol. 47, no. 15 , pp. 2835-2839, May 2008.

[10] D. Bo, et al., "Liquid-level sensor with a high-birefringence-fiber loop mirror," Appl. Opt., vol. 45, no. 30, pp. 7767-7771, Oct. 2006.

[11] O. Frazäo, B. Marques, P. Jorge, J. Baptista, and J. Santos, "High birefringence D-type fibre loop mirror used as refractometer," Sens. Actuators B, Chem., vol. 135, no. 1, pp. 108-111, Jan. 2008.

[12] K. J. Kim, S. H. Yeom, B. H. Kang, D. E. Kim, and S. W. Kang, "Micro-optic mach-zehnder interferometric sensor for UV detection using photochromic dye," Sensor Lett., vol. 9, no. 1, pp. 195-198, Feb. 2011.

[13] R. M. Andre, M. B. Marques, P. Roy, and O. Frazao, "Fiber loop mirror using a small core microstructured fiber for strain and temperature discrimination," IEEE Photon. Technol. Lett., vol. 22, no. 15, pp. 1120-1122, Apr. 1, 2010.

[14] S. Silva, et al., "Temperature and strain-independent curvature sensor based on a singlemode/multimode fiber optic structure," Meas. Sci. Technol., vol. 22, no. 8, p. 085201, Jun. 2011.

[15] R. M. Silva, A. Layeghi, M. I. Zibaii, H. Latifi, J. L. Santos, and O. Frazäo, "Theoretical and experimental results of high-birefringent fiber loop mirror with an output port probe," J. Lightw. Technol., vol. 30, no. 8, pp. 1032-1036, Apr. 15, 2012.

[16] M. Dahlem, J. L. Santos, L. A. Ferreira, and F. M. Araújo, "Passive interrogation of low-finesse Fabry-Pérot cavities using fiber Bragg gratings," IEEE Photon. Technol. Lett., vol. 13, no. 9, pp. 990-992, Sep. 2001.

[17] L. Amaral, O. Frazäo, J. Santos, and A. Lobo Ribeiro, "Fiber-optic inclinometer based on taper michelson interferometer," IEEE Sensors J., vol. 11, no. 9, pp. 1811-1814, Sep. 2011.

[18] R. M. Silva, A. B. Lobo Ribeiro, and O. Frazäo, "Temperatureindependent torsion sensor based on figure-of-eight fiber loop mirror," Photon. Sensors, vol. 10, no. 1, pp. 52-56, 2013. 